\title{
Statistical Emulation of Cardiac Mechanics: An Important Step towards a Clinical Decision Support System
}

\author{
Dirk Husmeier', Alan Lazarus', Umberto Noè², Vinny Davies ${ }^{3}$, Agnieszka Borowska1, \\ Benn Macdonald ${ }^{1}$, Hao Gao ${ }^{1}$, Colin Berry ${ }^{4}$ and Xiaoyu Luo ${ }^{1}$ \\ ${ }^{1}$ School of Mathematics and Statistics, University of Glasgow \\ University Place, Glasgow G12 8SQ, United Kingdom \\ dirk.husmeier@glasgow.ac.uk, a.lazarus.1@ research.gla.ac.uk, agnieszka.borowska@ glasgow.ac.uk, \\ benn.macdonal@glasgow.ac.uk, hao.gao@glasgow.ac.uk,xiaoyu.luo@glasgow.ac.uk \\ ${ }^{2}$ German Centre for Neurodegenerative Diseases (DZNE), \\ Sigmund-Freud-Straße 27, 53127 Bonn, Germany. \\ umberto.noe@dzne.de \\ ${ }^{3}$ School of Computing Science, University of Glasgow \\ 18 Lilybank Gardens, Glasgow G12 8RZ, United Kingdom \\ vinny.davies@glasgow.ac.uk \\ ${ }^{4}$ BHF Glasgow Cardiovascular Research Centre, University of Glasgow \\ University Place, Glasgow G12 8TA, United Kingdom \\ colin.berry@glasgow.ac.uk
}

\section{Extended Abstract}

In recent years we have witnessed substantial advances in the mathematical modelling of the biomechanical processes underlying the dynamics of the cardiac soft-tissue. In particular, in our recent work [1] we have demonstrated that the parameters underlying the biomechanical model have diagnostic value for prognosticating the risk of myocardial infarction (heart attack). However, determining the unknown parameters is computationally challenging, with the optimization process taking days or weeks to converge, even with a modern multi-core workstation. The primary reason for this is the high computational expense of simulating from the biomechanical model, which requires a numerical integration of the underlying partial differential equations with finite element discretization. This procedure has to be repeated hundreds or thousands of times during the iterative optimization of the material parameters. As a result of these high computational costs of simulating the biomechanical model, estimating myocardial properties using a process which uses this model as a simulator is not suitable for real-time clinical diagnosis.

A potential approach to overcome this problem is statistical emulation [2], which has recently been explored in the closely related contexts of cardiovascular fluid dynamics [3] and ventricular mechanics [4]. Emulation methods are far more computationally efficient as most of the computation can be done in advance, making the in-clinic diagnosis faster. With emulation approaches, we simulate a large number of samples at different parameter specifications in advance and use these simulations combined with an interpolation method to replace the computationally expensive simulator in the optimization procedure.

The work presented here is designed as a proof of concept study to assess the accuracy of alternative emulation strategies with Gaussian processes (GPs) [5] for learning the material properties of the left ventricle of the heart based on only noninvasive, in vivo magnetic resonance image (MRI) data. We compare different emulation strategies, loss functions and methods for dealing with high output dimensions. Our results can be summarized as follows.

Emulation strategies: We have compared output emulation, where the outputs of the mathematical model are emulated directly, with loss emulation, where we emulate the loss function that quantifies the agreement between the mathematical model and the data. Our simulations show that output emulation consistently outperforms loss emulation.

Gaussian process paradigm: For large data sets, it is not computationally feasible to train a GP, as the computational complexity is of the order of the third power of the data set size. Two paradigms for dealing with this issue have widely been used: sparse GPs [6] and local GPs [7]. Our results suggest that local GPs consistently outperform sparse GPs. 
Dealing with high-dimensional outputs: We show that modelling high-dimensional outputs with a multivariate-output GP gives better results than using several univariate output GPs, and we quantify the performance improvement achieved in this way.

Computational cost reduction: Most importantly, we have achieved a dramatic reduction of the computational complexity. While conventional parameter estimation based on numerical simulations from the cardiac mechanical model, following the approach described in [1], leads to computational costs in the order of weeks, the proposed emulation method reduces the computational complexity to the order of the quarter of an hour, while effectively maintaining the same level of accuracy.

This is an important step towards a clinical decision support system that can assist a clinical practitioner in real time.

\section{Acknowledgements}

This work was funded by the UK Engineering and Physical Sciences Research Council (EPSRC), grant number EP/N014642/1. Dirk Husmeier is supported by a grant from the Royal Society of Edinburgh, award number 62335.

\section{References}

[1] H. Gao, A. Aderhold, K. Mangion, X. Luo, D. Husmeier and C. Berry, "Changes and classification in myocardial contractile function in the left ventricle following acute myocardial infarction," Journal of The Royal Society Interface, vol.14, no.132:20170203, 2017.

[2] M.C. Kennedy, A. O'Hagan, "Bayesian calibration of computer models," Journal of the Royal Statistical Society, Series B (Statistical Methodology), vol. 63, pp. 425-464, 2001.

[3] A. Melis, R.H. Clayton, A. Marzo, "Bayesian sensitivity analysis of a 1d vascular model with Gaussian process emulators," International Journal for Numerical Methods in Biomechanical Engineering, vol. 33, no. 12, 2017.

[4] P.D. Achille, A. Harouni, S. Khamzin, O. Solovyova, J.J. Rice, V. Gurev, "Gaussian process regressions for inverse problems and parameter searches in models of ventricular mechanics," Frontiers in Physiology, vol. 9, doi: 10.3389/fphys.2018.01002, 2018.

[5] C.E. Rasmussen and C.K.I. Williams, Gaussian Processes for Machine Learning. MIT Press, 2006.

[6] M. Titsias, "Variational learning of inducing variables in sparse Gaussian processes," Proceedings of Machine Learning Research, vol.5, pp. 567-574, 2009.

[7] R.B. Gramacy and D.W. Apley, "Local Gaussian process approximation for large computer experiments," Journal of Computational and Graphical Statistics, vol. 24, no. 2, pp. 561-578, 2015. 